\title{
APPLYING A SIMULATION-BASED TOOL TO PRODUCTIVITY MANAGEMENT IN AN AUTOMOTIVE-PARTS INDUSTRY
}

\author{
Adrián Aguirre \\ Enrique Müller \\ Sebastián Seffino \\ Industrial Engineering Department (FIQ-UNL) \\ Santiago del Estero 2829 \\ Santa Fe, 3000, ARGENTINA
}

Carlos A. Méndez

INTEC (UNL -CONICET)

Güemes 3450

Santa Fe, 3000, ARGENTINA

\begin{abstract}
This work presents the development and application of an advanced modeling, simulation and optimization-based framework focused on the production process of a basic element of a internal combustion engine which is supplied by a leading factory in the Latin-American market. Lying on the concepts of the process-interaction approach, the principal components available in the discrete event simulation environment "SIMUL8" were used to achieve the best representation of this complex manufacturing system. Furthermore, advanced SIMUL8's Visual Logic tools were utilized for modeling specific design and operation features arising in the process under study. The developed tool provides a support system for making operative, tactical and strategic decisions, allowing the evaluation of possible scenarios ranging from different operation schemes to potential alternatives of investment. The principal aim of this work is to provide a systematic methodology to improve the productive capacity management, enhancing the process profitability and the degree of customer satisfaction.
\end{abstract}

\section{INTRODUCTION}

During the last years Argentina's economic growth was more than twice that of Bolivia, Brazil, Chile and Paraguay, becoming one of the leaders in the region, with major improvements in employment and income distribution and a decline in poverty levels. Within this economic positive context, the automotive industry has been directly affected with a significant rise in car sales and an increasing demand of car-parts. To take advantage of this opportunity, manufacturing plants related to the automotive business are being enforced to improve their process productive capacity. In this work we address the particular problem arising in a Company, dedicated to manufacture a high volume and variety of car-parts in Argentina. The major project goals aim at increasing the process productivity and the customer satisfaction.

In order to turn the company more profitable and competitive in a highly demanding market, it was observed the need to rely on effective computational tools to systematically analyze and evaluate the dynamic behavior of the process, considering different operative schemes and possible alternatives of investment.

Although the use of mixed-integer linear mathematical programming models (MILP) was considered in a preliminary stage of the project, the idea was quickly abandoned due to the lack of proper tools to manage large problem sizes, complex operational logic and different degrees of uncertainty in the problem data. In consequence, discrete events simulation methods were adopted to represent the whole real-world process in an integrated form. In this way, it was possible to clearly identify the major problem difficulties and challenges, providing validated answers to operative, tactical and strategic level requirements involving different scenarios and possible alternatives of investment. Similar applications based on simulation technology to the treatment of operative schemes and alternative of investment in the petrochemical industry can be found in Bacigalupo et al. (2006).

This paper is organized as follows. Section 2 introduces the company under study and defines the problem to be addressed. The proposed simulation-based methodology is presented in Section 3, describing the main steps followed in the model development, the system limitations, the embedded system logic and the user interfaces. Section 4 introduces different scenarios to give response to the problem challenges and defines the performance indicators to be considered along the study of the system. Finally, section 5 briefly describes and analyzes some preliminary results obtained by using the proposed simulation tool. 


\section{PROBLEM DEFINITION}

\subsection{The Company}

The main industrial activity of the Company is the manufacturing of a wide variety and a large volume of a basic element of the internal combustion engine, which are commercialized in different markets in a broad range of measures and applications, and under the most rigorous standards of quality. Currently, the company employs more than 50 people to reach a productive capacity of approximately 600.000 pieces monthly. Its present business volume and its integrated manufacturing system allow them to be among the three main car-parts factories in South America, reaching to export $52 \%$ of its production, almost totally to different Mercosur countries.

The manufacturing process comprises two different production lines and twelve processing stages depending on the main raw materials that are being used, i.e. bars or pipes. The company's policy is to meet the production orders arriving monthly at the time and the form that were previously agreed with the customers. To this end, the process is operated by selecting suitable small and medium production lots.

\subsection{Main Problems Issues}

At the present time, the constant rise of car-parts demand has motivated to the general managers of the company to improve the low process productivity associated with three main issues: a) low degree of customer satisfaction because of tardiness, b) large volume of work-in process inventory and, c) work-load unbalance of the manufacturing lines. The combination of these issues generates an inefficient utilization of the scarce production resources available to meet the higher demand. Based on the foregoing issues, it were defined a series of challenges directly focused on the actual needs of the company: a) to increase the productivity of manufacturing lines and, b) to respond in more agile and reliable form to the customer requirements.

In summary, the general goal is oriented towards optimizing the current productive capacity of the company in such a way that most of the deliveries can be met on time and in the right form. In addition, it is desired to be in position of quickly reacting to different commitments in the future with uncertain and variable conditions. Based on the current trend of the economy and the increasing car-parts demand, the company should be able to expand its productive capacity to at least 1.000 .000 pieces per month, which constitute the biggest challenge for this work.

\subsection{Solution Alternatives}

Different available solution alternatives were evaluated to cope with this problem. The use of an analytical method based on linear mathematical formulation tools (MILP models), which are broadly used to production optimization, was avoided because of the inherent computational and modeling limitations of these techniques. Also, the use of simple heuristic procedures was considered but they failed to handle the high complexity of the problem. Although these two approaches may be valid to generate optimal solutions for the system, they are not capable of effectively managing the random nature of many process variables (e.g. processing time distributions, material movement time distributions, etc.), the large number of resources involved and the complex operational rules to be considered. In order to overcome the limitations previously stated, it was decided to make use of a modern and userfriendly system analysis tool by developing an advanced computational process model lying on the notion of discrete event system simulation (Barcelo 1996, Banks et al. 2004, Koshnevis 1994)

\subsection{Advantages of Simulation-Based Methods}

The main advantage of the proposed computer-aided methodology is that it permits to systematically reproduce the complex company process in an abstract and integrated form, visualizing the dynamic behavior of its constitutive elements over time.

Production facilities (work centers), storage facilities (tails of waits, deposits of raw materials, intermediate storages and process storages), transports and materials movement devices (vehicles, bridge cranes, etc), external factors (variability in the arrival of the raw materials and demands), internal and unforeseen factors (changes in policies and operational rules, breaks, stops and maintenances of processing units in the production lines), and resources of the system (e.g. workers), were analyzed and modeled in detail. As result, a basic model was generated to discern the principal weaknesses and bottlenecks of the process, but it was also useful later for making decisions to enhance the current process performance.

\section{THE PROPOSED SIMULATION-BASED FRAMEWORK}

In order to formulate a precise computer-aided representation to the real-world manufacturing system described above, it was decided to make use of the simulation, visualization and analysis tool set provided in the discrete events simulation environment "SIMUL8" (Shalliker et al. 2002, Haige et al. 2001, Mc Gregor et al. 2004, SIMUL8 2004). The main characteristics of the proposed basic model are described in detail below. 


\subsection{Principal Model Features}

- Raw Materials: depending on the raw material being used in the process (pipes or bars), two alternative manufacturing lines were modeled. The main raw material characteristics are detailed in the Table 1 .

Table 1: Raw materials types

\begin{tabular}{rcl}
\hline Raw material & $\begin{array}{c}\text { Outside diameter } \\
{[\mathrm{mm}]}\end{array}$ & $\begin{array}{l}\text { Length } \\
{[\mathrm{meters}]}\end{array}$ \\
\hline bars & $22-60$ & $5.5-6$ \\
pipes & $16-28.6$ & 6 \\
\hline
\end{tabular}

- Product Family: three main final product types were identified according to the principal characteristics of the process (see Table 2).

Table 2: Final product types

\begin{tabular}{cc}
\hline Product family & Characteristics \\
\hline Small car-parts & Outside diameter $<25,4[\mathrm{~mm}]$ \\
Medium car-parts & Outside diameter $<38,1[\mathrm{~mm}]$ \\
Big car-parts & Outside diameter $<60,3[\mathrm{~mm}]$ \\
\hline
\end{tabular}

- Manufacturing process stages: The production sequence for every car-part family depends on the raw material being used. The general manufacturing stages are listed in Table 3 whereas the specific product recipes for every car-part family and raw material are shown in Table 4 and Figure 1. In turn, a partial size view of the in-progress SIMUL8 model can be seen in Figure 2. Additionally, the entire simulation model developed in the SIMUL8 environment can be found in Figure 3.

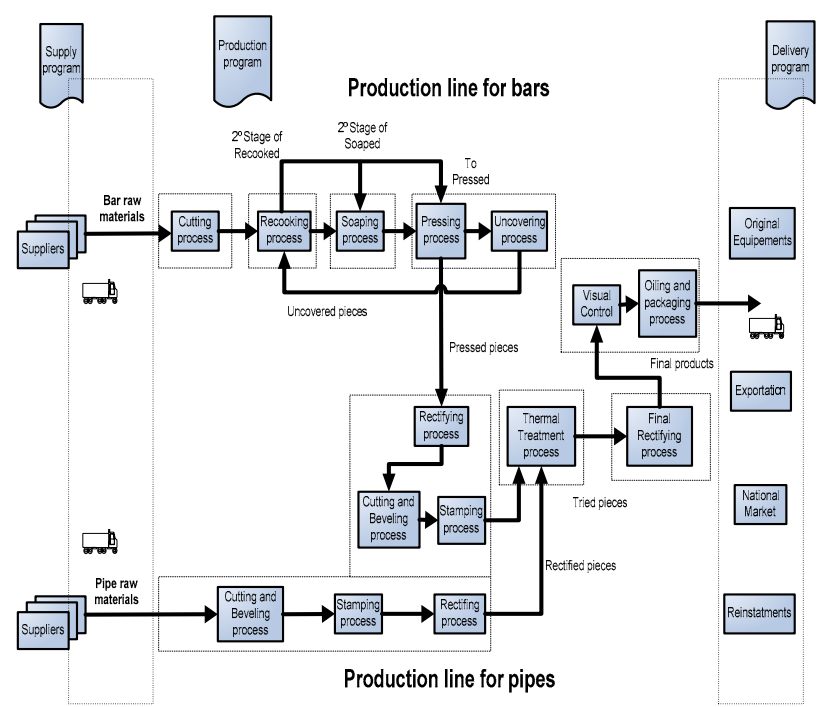

Figure 1: Global manufacturing system of the Company
Table 3: General manufacturing process stages

\begin{tabular}{|c|c|c|c|}
\hline $\begin{array}{l}\text { Process } \\
\text { stage }\end{array}$ & \multicolumn{2}{|c|}{ Description } & Available units \\
\hline 0 & \multicolumn{2}{|c|}{ Raw material reception } & - \\
\hline 1 & \multicolumn{2}{|c|}{ Bars cutting process } & $\begin{array}{l}3 \text { (cutting ma- } \\
\text { chines) }\end{array}$ \\
\hline 2 & \multicolumn{2}{|c|}{ Re-cooking process } & 3 (ovens) \\
\hline 3 & \multicolumn{2}{|c|}{ Soaping process } & 7 (vats) \\
\hline 4 & \multicolumn{2}{|c|}{ Pressing process } & 6 (press) \\
\hline 5 & \multicolumn{2}{|c|}{ Uncovering process } & 2 (press) \\
\hline 6 & \multicolumn{2}{|c|}{ Rectifying process } & 2 (machines) \\
\hline 7 & \multicolumn{2}{|c|}{$\begin{array}{l}\text { Cutting and beveling } \\
\text { process }\end{array}$} & 4 (machines) \\
\hline 8 & \multicolumn{2}{|c|}{$\begin{array}{c}\text { Thermal treatment } \\
\text { process }\end{array}$} & 12 (vats) \\
\hline 9 & \multicolumn{2}{|c|}{ Final rectifying process } & 4 (machines) \\
\hline 10 & \multicolumn{2}{|c|}{ Pipe cutting process } & $\begin{array}{l}1 \text { (cutting ma- } \\
\text { chine) }\end{array}$ \\
\hline 11 & \multicolumn{2}{|c|}{ Stamping process } & $\begin{array}{l}2 \text { (stamping ma- } \\
\text { chines) }\end{array}$ \\
\hline 12 & \multicolumn{2}{|c|}{ Final control } & (manual process) \\
\hline \multicolumn{4}{|c|}{ Table 4: Process sequence for each product family } \\
\hline Family & $\begin{array}{c}\text { Raw } \\
\text { material }\end{array}$ & \multicolumn{2}{|c|}{ Production sequence (stages) } \\
\hline $\begin{array}{r}\text { Small } \\
\text { car-parts }\end{array}$ & Bars & \multicolumn{2}{|c|}{$0-1-2-3-4-5-2-3-4-6-7-11-8-9-12$} \\
\hline $\begin{array}{l}\text { Medium } \\
\text { car-parts }\end{array}$ & Bars & \multicolumn{2}{|c|}{$0-1-2-3-4-5-2-3-4-6-7-8-9-12$} \\
\hline $\begin{array}{r}\text { Big } \\
\text { car-parts }\end{array}$ & Bars & \multicolumn{2}{|c|}{$0-1-2-3-4-5-2-3-4-6-7-8-9-12$} \\
\hline $\begin{array}{r}\text { Small } \\
\text { car-parts }\end{array}$ & Pipes & \multicolumn{2}{|c|}{$0-10-11-6-8-9-12$} \\
\hline $\begin{array}{l}\text { Medium } \\
\text { car-parts }\end{array}$ & Pipes & \multicolumn{2}{|c|}{$0-10-11-6-8-9-12$} \\
\hline
\end{tabular}

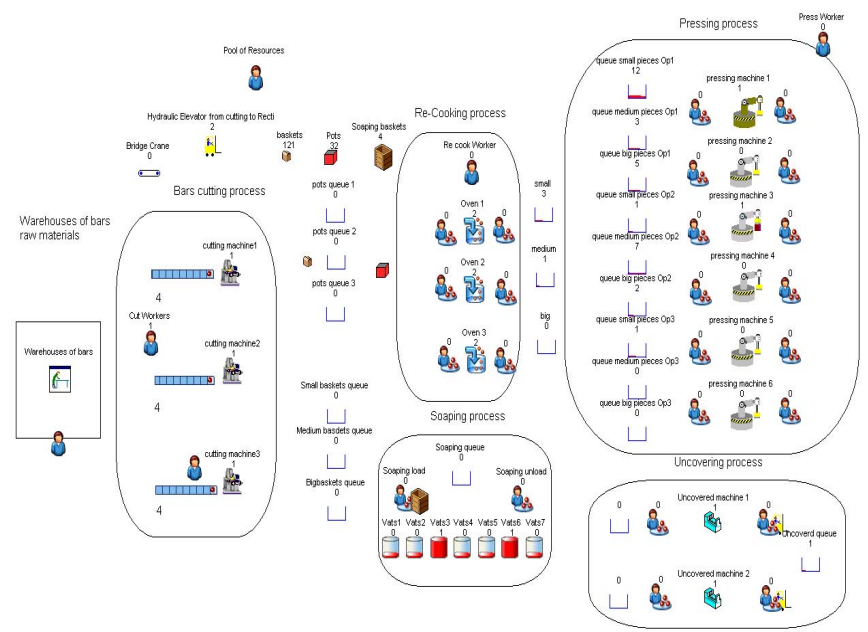

Figure 2: Partial size view of the in-progress SIMUL8 Model 


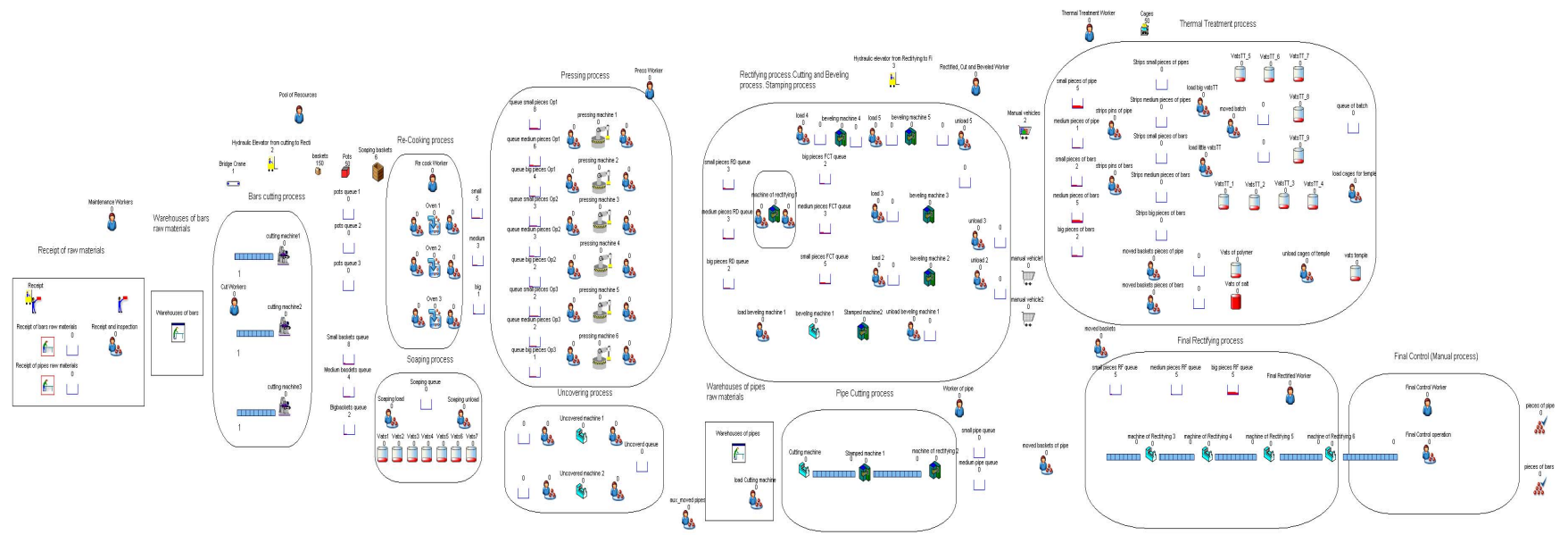

Figure 3: Computer-based representation of the entire manufacturing process model generated in the SIMUL8 environment

- Lot-sizing: Every order of product is divided into a number of batches for its processing and movement inside the factory. The maximum allowable batch size is defined in Table 5. This limitation comes mainly from the maximum feasible processing load and the available capacity of material handling resources.

Table 5: Maximum batch size for each product type Small car-part Medium car-part Big car-part 2500 1500 500

- $\quad$ Shift timetable: Three 8-hour work shifts are contemplated from Monday to Saturday. Also, an additional shift for Sunday is defined. The same ones are detailed in Table 6 .

Table 6: Shift timetable

\begin{tabular}{rccl}
\hline Shift & $\begin{array}{c}\text { Start time } \\
{[\mathrm{hrs}]}\end{array}$ & $\begin{array}{c}\text { End time } \\
{[\mathrm{hrs}]}\end{array}$ & Days \\
\hline Morning & $6: 00$ & $14: 00$ & Mon - Sat \\
Afternoon & $14: 00$ & $22: 00$ & Mon - Sat \\
Night & $22: 00$ & $6: 00$ & Mon - Fri \\
Sunday & $6: 00$ & $14: 00$ & Sun \\
\hline
\end{tabular}

- Resources of the System: Available resources for storage in transit, temporary storage and movement of materials are detailed in Table 7.
Table 7: Available production resources in the factory

\begin{tabular}{|c|c|c|}
\hline $\begin{array}{r}\text { Resource } \\
\text { name }\end{array}$ & Description & $\begin{array}{l}\text { Quantity } \\
\text { available }\end{array}$ \\
\hline Baskets & $\begin{array}{l}\text { Resource for the storage } \\
\text { in process }\end{array}$ & 150 \\
\hline Pots & $\begin{array}{l}\text { Resource for the storage } \\
\text { in process }\end{array}$ & 50 \\
\hline $\begin{array}{r}\text { Soaping } \\
\text { baskets }\end{array}$ & $\begin{array}{l}\text { Resource for the storage } \\
\text { in process }\end{array}$ & 6 \\
\hline Cages & $\begin{array}{l}\text { Resource for the storage } \\
\text { in process }\end{array}$ & 50 \\
\hline $\begin{array}{r}\text { Hydraulic } \\
\text { elevators }\end{array}$ & $\begin{array}{l}\text { Manual vehicle for mate- } \\
\text { rials handling }\end{array}$ & 5 \\
\hline $\begin{array}{l}\text { Bridge } \\
\text { crane }\end{array}$ & $\begin{array}{l}\text { Device for materials han- } \\
\text { dling }\end{array}$ & 1 \\
\hline $\begin{array}{r}\text { Manual } \\
\text { Vehicles }\end{array}$ & $\begin{array}{l}\text { Manual vehicle for mate- } \\
\text { rials handling }\end{array}$ & 2 \\
\hline Hoist & $\begin{array}{l}\text { Vehicle for materials } \\
\text { handling }\end{array}$ & 1 \\
\hline Conveyors & $\begin{array}{l}\text { Device for materials han- } \\
\text { dling }\end{array}$ & 10 \\
\hline
\end{tabular}

- Available manpower: The workers were modeled as an additional manufacturing resource in the production system with a dynamic availability over time. The allocation of workers to processing stages depends directly on the work shift and it is detailed in Table 8. In addition, programmed and non-programmed breaks and absenteeism can be easily incorporated in the simulation model. 
Table 8: Dynamic manpower availability in the factory

\begin{tabular}{rcclll}
\hline name & Processing stages & \multicolumn{4}{c}{ Worker availability for each work shift } \\
(workers) & & Morning & Afternoon & Night & Sunday \\
\hline Cut worker of bars & 1 & 1 & 1 & 1 & 0 \\
Re-cook worker & $2-3$ & 1 & 1 & 1 & 1 \\
Maintenance worker & all & 2 & 2 & 2 & 2 \\
Press worker & $4-5$ & 4 & 4 & 3 & 3 \\
$\begin{array}{r}\text { Rectified, cut } \\
\text { and beveled worker }\end{array}$ & $6-7$ & 3 & 4 & 3 & 0 \\
Thermal treatment & 8 & 4 & 4 & & 0 \\
worker & & & & 0 & 0 \\
Final rectified worker & 9 & 4 & 4 & 0 & 0 \\
Final control worker & 12 & 5 & 5 & 0 & 0 \\
Worker of pipes & $10-11-6-8-9$ & 1 & 1 & & \\
\hline
\end{tabular}

- Data modeling: The processing time of the principal units was modeled using a normal probability distribution, which depends on the product type, the operation stage and the characteristic of the raw material that is being used (i.e. outside diameter). The validated parameters of the distribution were obtained after conducting the corresponding statistics studies. Likewise, two different setup times were modeled using deterministic values. The first one depends on the changes in product type and the current operation process. The second one was modeled for changes in process operations, i.e. for multipurpose units. These conditions were carefully programmed by using SIMUL8's Visual Logic functions (VL) for each distribution. Also, uniform time distributions for materials movements and loading and unloading of materials in process were employed.

- Simulation clock: Before running the simulation model, two parameters related to the simulation clock need to be defined, i.e. the Warm Up Period and the Result Collect Period. In this case, although the Warm up Period was set to zero, proper initial inventories were introduced in order to imitate the usual process conditions. In turn, an appropriate Result Collect Period was selected depending on the type of analysis to be performed. A weekly time (168 hours) was employed for model validation purposes. In contrast, a monthly time (720 hours) was used to validate and conduct studies regarding the dynamic model behavior, taking into account different features such as the operative rules and policies, the average in-process inventory, percentages of utilization of workers, work centers and resources of the system.

\subsection{Model Development and Operative Rules}

Through the development of the simulation model, it were identified all the manufacturing restrictions and operational rules to be considered. These types of restrictions due to operative intrinsic factors (e.g. maximum batch sizes for every car-part family, available manpower in each work shift, process operations and manufacturing stages allowed in each work center, etc.) as well as external factors (e.g. raw material shortfalls, demand variability, etc.).

These features were directly modeled by using the Visual Logic function (VL) available in the SIMUL8 software, based on the occurrence of different system events. An illustrative example for the embedded logic behind the order priority definition is shown Figure 4. Other actual manufacturing restrictions and operating rules are described below.

1. Production batch sizes are based on the maximum capacity defined for every product type. The selected size is maintained through the entire production process, comprising processing stages and material movements). The material movement task is carried out for every batch. This activity requires a resource for storage in transit (e.g. one basket), the corresponding resource for material movement (e.g. an hydraulic elevator) and the allocated manpower (e.g. one of the press workers).

2. The material movement activities have higher priority than any other processing activity.

3. Processing units are modeled as unary resources, i.e. batches are processed one by one in every work center, except in certain centers like ovens. In these manufacturing resources, two batches must be processed simultaneously. Each oven can perform two operations $\left(1^{\text {st }}\right.$ re-cooked and $2^{\text {nd }}$ re-cooked). In every work load, it can be 
mixed batches of different product types but not batches of different operations.

4. Some facilities can perform several operations on different product types (multipurpose units). For example, some presses are able to carry out three operations for every product type whereas the ovens can perform two operations $\left(1^{\text {st }}\right.$ re-cooked and $2^{\text {nd }}$ re-cooked). Each kind of operation has a different processing time and setup time distributions. In consequence, tasks allocations for every product type at every work center are carefully managed in such a way that setup times can be minimized.

5. Raw materials (pipes and bars) are classified into several subtypes depending on the outside diameter. In order produce a particular order, it must be guaranteed the use of those subtypes (pipes or bars) in which the outside diameter is equal or larger than the diameter of the order. In case that at some time there is no availability of raw materials satisfying the outside diameter, the beginning of order must delayed up to the reception of the corresponding supply of material.

6. In every manufacturing stage, the processing of production orders is arranged depending on the predefined commercial priority.

7. In the case that all resources for material storage were totally busy, the model does not allow the assignment of resources to certain work centers in order to avoid any resource blockade.

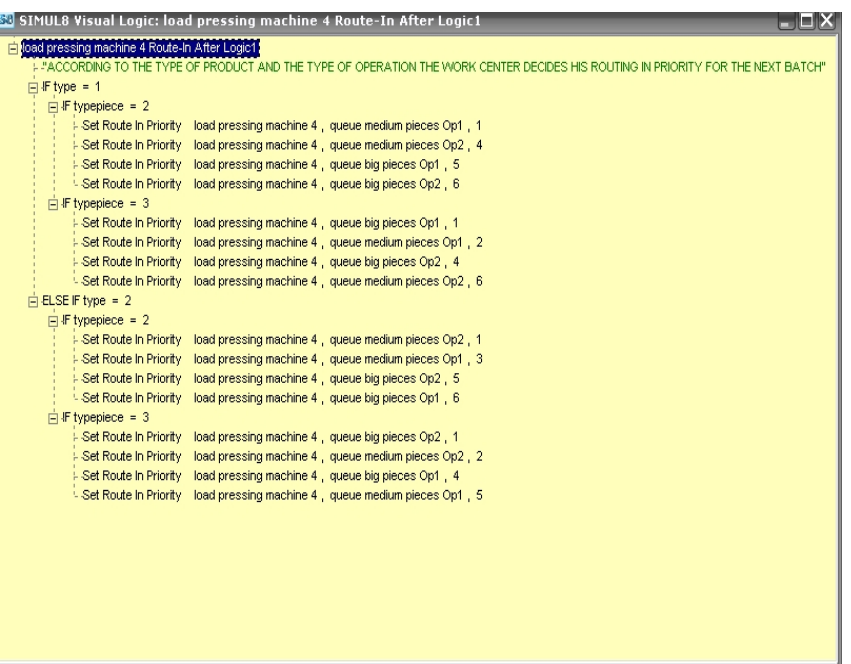

Figure 4. Representation of operative rules through SIMUL8's Visual Logic programming

In the same way, a series of dialogs and menus were developed by using Visual Basic tools in order to provide an interactive and user-friendly model interface. These tools allow the user to enter all the problem data and also to propose changes to the design and operative conditions of the production system. Moreover, it provides an effective way to analyze and study model results during the simulation run (see Figures 5 to 8).

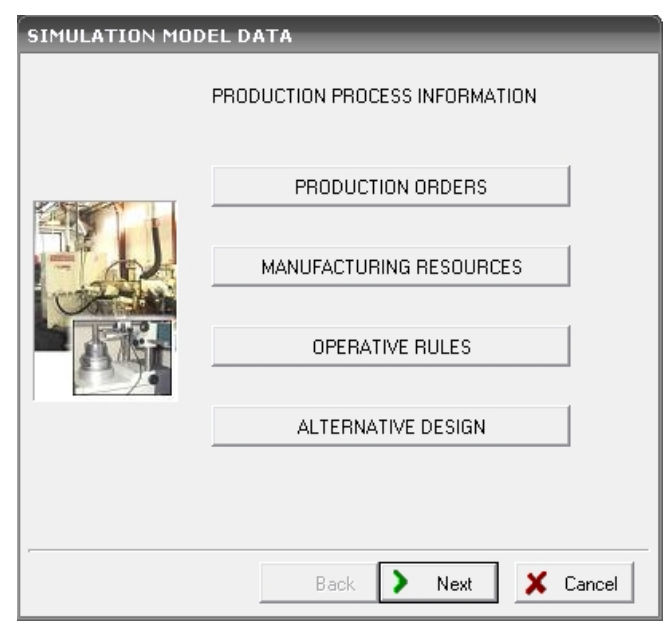

Figure 5: Principal menu

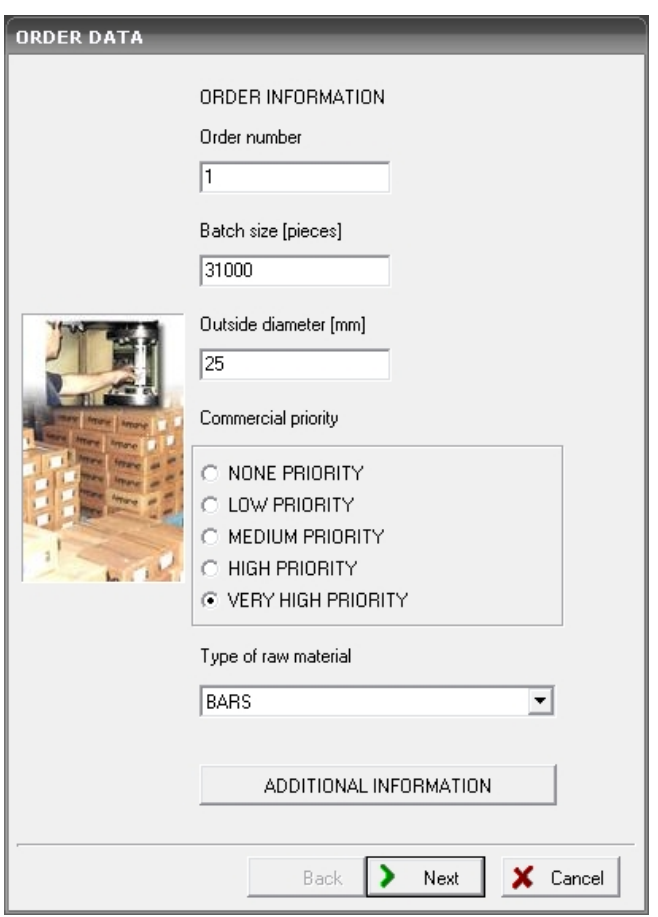

Figure 6: Interface order data

Once the problem data was loaded in the simulator interfaces and the restrictions and operational rules were correctly programmed, it was possible to perform the validation of the simulation model in order to guarantee that the model imitates the current process conditions. Also, it was carefully observed if the model was slightly sensitive to superfluous changes in the system. 
To do this, it was defined and analyzed a set of measures and indicators of performance and effectiveness in order to corroborate the correct computational representation of the real system. Total production volume, fulfilment of production goals, average amount of in-process inventory in every manufacturing stage were some of the system output variables that were analyzed in detail.

The study of the dynamic behavior of certain system resources, such as machines, storage resources, workers and in-process inventory levels in each stage, was carried out using different SIMUL8 visual tools oriented to system output analysis (see Figures 7 and 8).

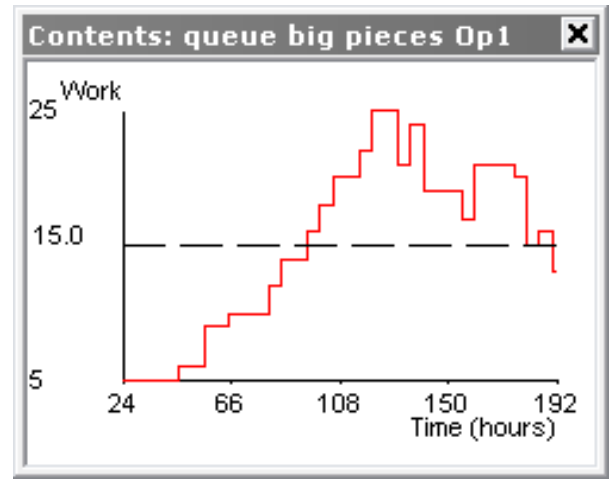

Figure 7: Dynamic evolution of the in-process inventory level of "queue big pieces Op1" in the Pressing Stage.

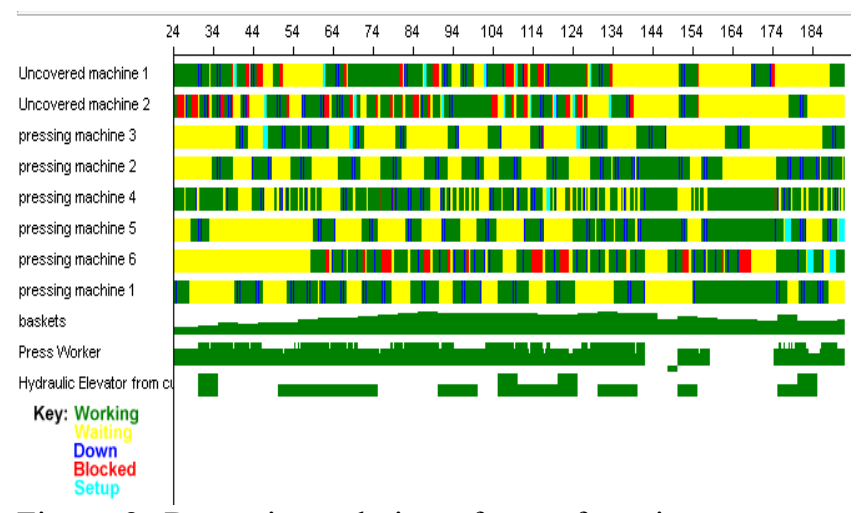

Figure 8: Dynamic evolution of manufacturing resource conditions along the simulation run

On the basis of the simulation results, it was possible to conclude that the development of the analyzed and validated basic model was useful in a preliminary project stage to identify the possible weak points in the manufacturing lines and to visualize the complex dynamic of global process.

This basic model is the principal tool for the study and analysis of alternative system solutions. In addition, it represents a suitable model to evaluate future decisions that could improve the proposed criterion as well as to propose better solutions that help to increase the current process performance.

\section{CONDUCTING SIMULATION STUDIES}

After identifying the major operational issues in the process, it was proposed a series of changes related to design and operative features, which a priori could increase the productive capacity of the company and fix the weak points of the process.

To do this, alternative scenarios are to be generated, identifying the values of the factors that are considered to be more relevant. Some of them are explicitly defined below.

- Increasing the available capacity of the resources associated with the material movement.

- Increasing the existing manufacturing resources in the likely bottlenecks.

- Reassignment and dynamic re-scheduling of available workers and resources of the system.

- Increasing the number of machines in the principal process stages.

- Modification of some basic operative rules: Order sequencing in shared manufacturing resources (e.g. minimizing the number of setup times for work center, pre-assignment of tasks and products to be processing in every work center, modification in the processing sequence, etc.).

\subsection{Measures of Effectiveness}

Our primary measures of effectiveness and performances are the following ones:

- Production volume in every work center (carparts/Week or Pieces/Month).

- Percentages of utilization of workers, work centers and resources of the system (see Figures 9 and 10).

- Production volume per type of product (carparts/Week or Pieces/Month). (see Figure 10)

- Average in-process inventory in every stage

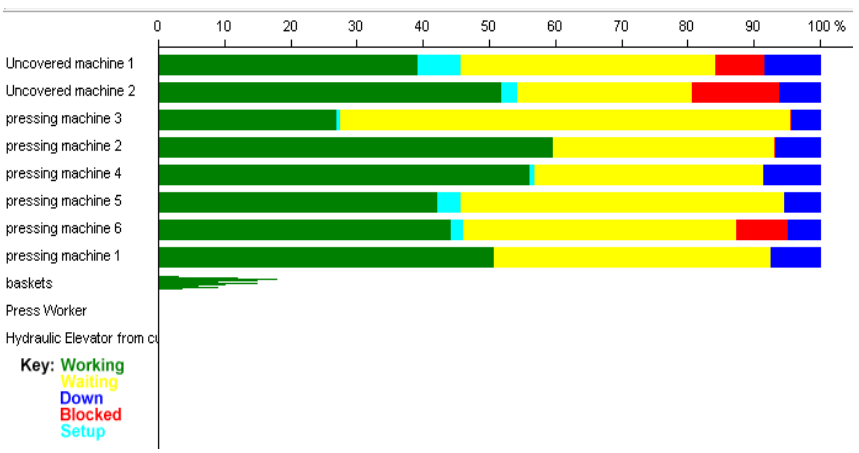

Figure 9: Summary of resource conditions along the simulation run 


\section{RESULTS}

This section presents some results of interest related to the main operational difficulties and weak points that were observed in the manufacturing process. The analysis of the results obtained from the simulation model output aims at providing some systematic rules and suggestions that allow the company to reach the major project goals.

The first step of the simulation-based study was mainly focused on identifying the possible bottlenecks of the process. After analyzing the principal measures of effectiveness of the system, which are obtained from several runs of the validated simulation model assuming different production orders and operative schemes, it was possible to determine that the critical process operations, i.e. the production bottlenecks, were mainly related to the processing stages of re-cooking, pressing and uncovering (see Figures 1 and 10).

\section{Results}

from most recent run only.

\begin{tabular}{lll}
\hline Cut Workers & Utilization \% & 7.75 \\
Re cook Worker & Utilization \% & 57.27 \\
Press Worker & Utilization \% & 95.09 \\
Rectified, Cut and Beveled Wor & Utilization \% & 61.69 \\
Worker of pipe & Utilization \% & 100.00 \\
Thermal Treatment Worker & Utilization \% & 27.26 \\
Final Rectified Worker & Utilization \% & 22.67 \\
baskets & Utilization \% & 33.28 \\
Cages & Utilization \% & 5.50 \\
Pots & Utilization \% & 36.46 \\
Soaping baskets & Utilization \% & 16.96 \\
Hydraulic Elevator from cutting & Utilization \% & 35.48 \\
Bridge Crane & Utilization \% & 47.36 \\
Manual vehicles & Utilization \% & 0.94 \\
Hydraulic elevator from Rectify & Utilization \% & 5.01 \\
pieces of pipe & Number Completed & 70000.00 \\
pieces of bars & Number Completed & 73340.00
\end{tabular}

Figure 10: Utilization of workers, work centers and other resources of the system. Production volume per type of product (car-parts/Week).

These critical stages comprise several processing units that can perform different operations on a variety of product families, i.e. they work as multipurpose units. Based on this feature, effective operative rules were identified to make the most convenient allocation of opera- tions to these critical machines. The main goal of this task is to balance the work load in previous and next stages. The selection of the best operative rules was achieved by analyzing different storage policies, the average inprocess inventory in the critical stages, the cycle time of every product type and the best mix of production.

Therefore, the production capacity of the entire manufacturing process as well as their individual processing stages was also carefully studied. The critical operations enforcing hard upper limits on the whole production rate were explicitly identified. To carry out this study, these resources were analyzed considering the availability of hours-man and/or hours-machines per month for the current operative schemes. The maximum production capacities were defined in terms of pieces per month that could be performed in every operation, taking into account the accomplishment of the company production targets. Based on the use of these criterion, it was possible to distinguish which operations were enforcing hard limits on the company production rate. These operations were pressing, cutting and beveling and thermal treatment.

These estimations were certainly useful not only to find out the maximum theoretical capacity of the system under the validated operative conditions but also to determine the most convenient assignment of manufacturing operations to the available machines in order to avoid unnecessary setup times.

The operational rules previously described were embedded in the simulation model by using the Visual Logic tools provided in the Simul8 package. These were mainly oriented to properly determine; a) lot sizes to be processed for every family of product, b) critical quantities of inprocess inventory in each stage which determine the change of operations in the machines and, c) efficient assignment of manufacturing resources to the corresponding machines.

Some general conclusions were drawn by using the simulation-based results, which mainly aims at improving the current productive capacity of the whole system. Some of them are briefly stated below:

- It was suggested to revise and modify the policies used for handling production orders. Particularly, it was recommended to employ the simulation model to estimate the quantities and due dates that can be promised to customers.

- It was proposed to re-classify and revise the sales policies in order to increase the reliability and the degree of satisfaction of clients.

- It was recommended to continue performing a more exhaustive analysis of the process by means of developed simulation model before making significant investments to replace and/or upgrade the current processing units. It was systematically demonstrated through the simulation study that investing on certain non-critical opera- 
tions may not have a positive effect on the system productive capacity.

- Finally, it was recommended to the company to use the simulation tool to conduct different tests on alternative scenarios in order to get more experience and knowledge about the inherent dynamic process behavior. This information should be very useful to efficiently manage the unforeseen events typically happening in this highly dynamic industrial environment.

\section{CONCLUSIONS}

This work presented the major steps followed for the development and the application of an advanced modeling, simulation and optimization tool aiming at productive capacity management of a car-parts factory. The proposed framework allows to easily represent and validate the existing operative schemes in the company in order to determine the current weak points and bottlenecks in the manufacturing lines that need to be improved. In addition, the developed tool can be used to analyze, test and predict the dynamic behavior of the system after introducing modifications in the production schemes and/or possible alternatives of investment.

\section{ACKNOWLEDGMENTS}

The authors are thankful for financial support from FONCYT-ANPCyT under Grant PICT-01837, from CONICET under Grant PIP-5729 and from Universidad Nacional del Litoral under CAI+D 003-13.

\section{REFERENCES}

Bacigalupo, C.M, C.A. Gratti, A.O. Martinez, L.A. Franzece, M.M. Fiorini, and D.P. Paz. 2006. Simulación computacional de la cadena logística de la refinería San Lorenzo. Revista del Instituto Argentino del Petróleo. Available from: $<$ http://www.paragontech.com.ar $>$ [accessed March $1,2008]$.

Banks, J., J. Carson, B. Nelson, and D. Nicol. 2004. Discrete event system simulation. 4th ed. Prentice-Hall.

Barceló, J. 1996. Simulación de Sistemas discretos. Isdefe: Edison.

Haige, J.W., and K.N. Paige. 2001. Learning SIMUL8: The Complete Guide. Plain Vu: Bellingham, WA.

Koshnevis, B. 1994. Discrete systems simulation. McGraw-Hill.

Law, M., and W. Kelton. 1991. Simulation modeling and analysis. McGraw-Hill.

Mc Gregor, D.W., and M.J. Cain. 2004. An introduction to SIMUL8. Department of Management, College of
Business and Economics, University of Canterbury, New Zealand.

Shalliker, J., and C. Ricketts. 2002. An Introduction to SIMUL8. Release nine. School of Mathematics and Statistics, University of Plymouth.

SIMUL8: Manual and User Guide. 2004. Visual Thinking International: Mississauga, Ontario Canada.

\section{AUTHOR BIOGRAPHY}

CARLOS A. MENDEZ is a Titular Professor of Industrial Engineering at The Universidad Nacional del Litoral in Argentina as well as an Adjoint Researcher of the National Scientific and Technical Research Council (CONICET) in the area of Process Systems Engineering. Dr. Méndez obtained his B.S. degree from The Universidad Tecnológica Nacional in Argentina and his $\mathrm{PhD}$ in Engineering from The Universidad Nacional del Litoral in Argentina. He has published over 70 refereed journal articles, book chapters, and conference papers. His research and teaching interests include production planning and scheduling, optimization, logistics and discrete-event simulation. 